\title{
African Nightshade (Solanum scabrum Mill.): Impact of Cultivation and Plant Processing on Its Health Promoting Potential as Determined in a Human Liver Cell Model
}

\author{
Grace Akinyi Odongo ${ }^{1,2}$, Nina Schlotz ${ }^{1}$, Susanne Baldermann ${ }^{3,4}$, Susanne Neugart ${ }^{3}$, \\ Susanne Huyskens-Keil ${ }^{5}$, Benard Ngwene ${ }^{3}$, Bernhard Trierweiler ${ }^{6}$, Monika Schreiner ${ }^{3}$ (D) \\ and Evelyn Lamy ${ }^{1, *}$
}

1 Molecular Preventive Medicine, Institute for Infection Prevention and Hospital Epidemiology, University Medical Center and Faculty of Medicine, University of Freiburg, Breisacher Strasse 115b, 79106 Freiburg, Germany; grace.odongo@uniklinik-freiburg.de (G.A.O.); nina.schlotz@uni-konstanz.de (N.S.)

2 Institute of Food Chemistry, Hamburg School of Food Science, University of Hamburg, 20146 Hamburg, Germany

3 Leibniz Institute of Vegetable and Ornamental Crops, Theodor-Echtermeyer-Weg 1, 14979 Großbeeren, Germany; Baldermann@igzev.de (S.B.); neugart@igzev.de (S.N.); Benard.Ngwene@agcocorp.com (B.N.); Schreiner@igzev.de (M.S.)

4 Department of Food Chemistry, Institute of Nutritional Science, University of Potsdam, Arthur-Scheunert-Allee 114-116, 14558 Nuthetal, Germany

5 Division Urban Plant Ecophysiology, Faculty of Life Science, Humboldt University Berlin, Lentzeallee 55/57, 14195 Berlin, Germany; susanne.huyskens@hu-berlin.de

6 Max Rubner-Institut, Federal Research Centre for Nutrition and Food, Institute of Safety and Quality of Fruits and Vegetables, Haid-und-Neu Strasse 9, D-76131 Karlsruhe, Germany; bernhard.trierweiler@mri.bund.de

* Correspondence: evelyn.lamy@uniklinik-freiburg.de; Tel.: +49-76-127082150

Received: 20 September 2018; Accepted: 10 October 2018; Published: 17 October 2018

\begin{abstract}
Plant cultivation and processing may impact nutrient and phytochemical content of vegetables. The present study aimed at determining the influence of cultivation and processing on the health promoting capacity of African nightshade (Solanum scabrum Mill.) leaves, an indigenous vegetable, rich in nutrients and phytochemicals. Anti-genotoxicity against the human liver carcinogen aflatoxin $\mathrm{B} 1\left(\mathrm{AFB}_{1}\right)$ as determined by the comet assay and radical oxygen species (ROS) scavenging capacity of ethanolic and aqueous extracts were investigated in human derived liver (HepG2) cells. ROS scavenging activity was assessed using electron paramagnetic spin resonance and quantification of ARE/Nrf2 mediated gene expression. The cultivation was done under different environmental conditions. The processing included fermentation and cooking; postharvest ultraviolet irradiation (UV-C) treatment was also investigated. Overall, S. scabrum extracts showed strong health promoting potential, the highest potential was observed with the fermented extract, which showed a $60 \%$ reduction of $\mathrm{AFB}_{1}$ induced DNA damage and a $38 \%$ reduction in $\mathrm{FeSO}_{4}$ induced oxidative stress. The content of total polyphenols, carotenoids and chlorophylls was indeed affected by cultivation and processing. Based on the present in vitro findings consumption of $S$. scabrum leaves could be further encouraged, preferentially after cooking or fermentation of the plant.
\end{abstract}

Keywords: aflatoxin B1; African indigenous vegetables; anti-genotoxicity; anti-oxidant activity; cancer chemoprevention; Solanaceae 


\section{Introduction}

The broad-leafed African nightshade (Solanum scabrum Mill.), locally known as mnavu in Swahili language, is widely used in West, Central and East Africa where it is cultivated as leafy vegetable [1]. The leaves are rich in nutrients, especially proteins, iron, ascorbic acid and riboflavin [2,3] which makes it a very important nutritional source for poor people. During the last years, a rising trend in the use of this crop can be observed as local people become aware of its proclaimed health benefits. A variety of health promoting, bioactive phytochemicals such as phenolic compounds, carotenoids and chlorophylls [2,3] have been identified recently in S. scabrum, which could be the reason for its gained popularity in the urban areas but also help explain its long use in traditional medicine. However, it is still unclear how these contribute to human nutrition and health, since scientific evidence on the health promoting activities of $S$. cabrum is scarce.

With increasing demand for this crop in markets, farmers in peri-urban areas have consequently increased its production. The leaves of Solanum species are more consumed than the fruits since these can contain high amounts of anti-nutrients such as solanaceous glycoalkaloids like solasodine [4]. For the leaves, this is normally less [4] but they are cooked or fermented with milk to reduce these bitter tasting compounds [4]. Glycoalkaloids have not been detected in S. scabrum but in S. nigrum and S. villosum [4]. Processing conditions including cooking or fermentation, have been reported to impact the phytochemical content of vegetables and fruits [5-9]. Further, this is true for postharvest treatment procedures such as drying or UV-C treatment and also depends on the geographical region [3].

Based on this background, the present study focused on the impact of plant processing on the health promoting, cancer preventive bioactivity of S. scabrum leaves. Anti-genotoxicity in terms of DNA protection against aflatoxin B1 and reactive oxygen species (ROS) scavenging capacity was determined using a metabolically competent human liver cell (HepG2) model. The phytochemical content of the differently cultivated and processed S. scabrum was also determined to link bioactivity with the phytochemicals in the plant.

\section{Materials and Methods}

\subsection{Chemicals}

Absolute ethanol (EtOH), hydrochloric acid (37\%), trypan blue, aflatoxin B1 (purity $\geq 98 \%$ ), menadione, ethidium bromide and DEAE-Sephadex A-25 were purchased from Sigma-Aldrich Chemie GmbH (Taufkirchen, Germany). Krebs HEPES buffer (KHB), deferoxamine methanesulfonate (DFO), diethyldithiocarbamic acid sodium (DETC) and 1-hydroxy-3-methoxycarbonyl-2,2,5,5-tetramethylpyrrolidine (CMH) were purchased from Noxygen Science Transfer \& Diagnostics GmbH (Elzach, Germany). L-glutamine, penicillin and streptomycin were purchased from Invitrogen ${ }^{\mathrm{TM}}$ Fisher Scientific $\mathrm{GmbH}$ (Schwerte, Germany). Dulbecco's modified Eagle's medium (DMEM), foetal calf serum (FCS), trypsin $10^{*}(25 \mathrm{mg} / \mathrm{mL})$, trypsin-EDTA $10^{*}(5$ $\mathrm{mg} / \mathrm{mL}$ and $2.2 \mathrm{mg} / \mathrm{mL}$ ) and phosphate buffered saline (PBS, without $\mathrm{Ca}$ and $\mathrm{Mg}$ ) were purchased from Gibco ${ }^{\mathrm{TM}}$, Life Technologies GmbH (Darmstadt, Germany). Triton-X 100 was from Carl Roth GmbH \& Co. KG (Karlsruhe, Germany) and dimethyl sulfoxide (DMSO; purity > 99\%) was purchased from Applichem GmbH (Darmstadt, Germany). Low melting point agarose (LMPA) and normal melting point agarose (NMPA) were purchased from Serva GmbH (Heidelberg, Germany).

The following chemicals and reagents were used for chemical analyses: Methanol (99.95\%), ammonium acetate (Carl Roth GmbH \& Co. KG, Karlsruhe, Germany); tetrahydrofuran (99.7\%; VWR International GmbH, Darmstadt, Germany); methyl tert-butyl ether (99.8\%; Chemsolute, Th. Geyer GmbH \& Co. KG, Renningen, Germany); dichloromethane (99.9\%), isopropanol (99.95\%) and zeaxanthin were purchased from Carote Nature $\mathrm{GmbH}$ (Ostermundigen, Switzerland); $\beta$-carotene, lutein and chlorophylls a \& b were from Sigma-Aldrich Chemie GmbH (Taufkirchen, Germany). Chlorogenic acid, quercetin 3-O-glucoside, kaempferol 3-O-glucoside and isorhamnetin-3-O-glucoside were from Carl Roth $\mathrm{GmbH}$ and Co. KG (Karlsruhe, Germany). Ferrous sulphate heptahydrate 
$\left(\mathrm{FeSO}_{4} \cdot 7 \mathrm{H}_{2} \mathrm{O}\right)$ was purchased from Merck Chemicals $\mathrm{GmbH}$ (Darmstadt, Germany). Arylsulfatase, isolated from Helix pomatia, was purchased from Roche Diagnostics GmbH (Mannheim, Germany).

\subsection{Solanum scabrum}

Seeds of S. scabrum were provided by the World Vegetable Centre (AVRDC) and cultivated at four different locations; the Max Rubner Institute, Federal Research Institute of Nutrition and Food, Karlsruhe, Germany (location A; 49 $0^{\prime} 50.976^{\prime \prime} N 8^{\circ} 25^{\prime} 35.832^{\prime \prime}$ E and altitude $116 \mathrm{~m}$ above sea level), the Leibniz Institute of Vegetable and Ornamental Crops Großbeeren (IGZ) Großbeeren, Germany (location B; $52^{\circ}$ latitude North, $13^{\circ}$ longitude East and altitude $43 \mathrm{~m}$ above sea level), Humboldt University, Berlin, Germany (location C; $52^{\circ}$ latitude North, $13^{\circ}$ longitude East and altitude $43 \mathrm{~m}$ above sea level) and at Jomo Kenyatta University of Agriculture and Technology (JKUAT), Nairobi, Kenya (location D; latitude $1^{\circ} 18^{\prime} \mathrm{N}$; longitude $37^{\circ} 12^{\prime} \mathrm{N}$ ).

Growth conditions at the different location was as follows; at location A, S. scabrum was cultivated using peat-based substrate (Gramoflor) with the following specifications: $\mathrm{pH}\left(\mathrm{CaCl}_{2}\right)$ 5.8; $\mathrm{N}\left(\mathrm{CaCl}_{2}-\mathrm{mg} / \mathrm{L}\right) 140 ; \mathrm{P}_{2} \mathrm{O}_{5}(\mathrm{Cal}-\mathrm{mg} / \mathrm{L}) 160 ; \mathrm{K}_{2} \mathrm{O}(\mathrm{Cal}-\mathrm{mg} / \mathrm{L}) 180$. The plants were grown in a climatic chamber for four weeks at day/night temperatures of $25 / 20^{\circ} \mathrm{C}$, relative humidity of $40 / 70 \%$ and light for $12.5 \mathrm{~h}$. After four weeks, plants were transferred to the greenhouse and kept there under ambient temperature (according to seasonal influences up to $40{ }^{\circ} \mathrm{C}$ in the summer) and without humidity control for three weeks.

At location B, seeds of S. scabrum were sown in $5 \mathrm{~L}$ plastic pots containing standard potting substrate (Einheitserdewerke Werkverband e.V., Sinntal-Altengronau, Germany) with the following specifications: $\mathrm{pH}\left(\mathrm{CaCl}_{2}\right)$ 5.8; $\mathrm{KCl}(\mathrm{g} / \mathrm{L}) 2 ; \mathrm{N}\left(\mathrm{CaCl}_{2}-\mathrm{mg} / \mathrm{L}\right) 340 ; \mathrm{P}_{2} \mathrm{O}_{5}(\mathrm{Cal}-\mathrm{mg} / \mathrm{L}) 380 ; \mathrm{K}_{2} \mathrm{O}$ (Cal-mg/L) 420. The plants were grown in a greenhouse for seven weeks after germination with sufficient irrigation. During this period, average day/night temperature was $21 / 18.6^{\circ} \mathrm{C}$ respectively with an average air humidity of $54 \%$. Additional cultivation experiment was done by growing some plants in an open green house. During harvest, fully developed leaves were collected in triplicate, each replicate consisting of $200 \mathrm{~g}$ of fresh leave material pooled from about ten plants. The leaves were then either immediately freeze-dried (control), or processed for further treatments (fermentation or thermal processing or UV treatment). At location C, the seeds of S. scabrum were grown under greenhouse conditions at the experimental station in Berlin-Dahlem, with the temperature, relative air humidity and light intensity during the experiments continuously recorded. Eight weeks after sowing, leaves were harvested and immediately subjected to UV-C treatments.

At Location D, seeds of $S$. scabrum were sown directly on an experimental field plot with a spacing of $20 \times 30 \mathrm{~cm}$. The soil is described as a clay soil with a pH of about 5.2. Mean annual rainfall in Juja, Nairobi, is about $1500 \mathrm{~mm}$ and temperature of about $20^{\circ} \mathrm{C}$ averaged the years $2000-2012$ according to World weather online (2016). Before planting, the plot was supplied with $2 \mathrm{~kg} / \mathrm{m}^{2}$ well-decomposed manure and $5 \mathrm{~g}$ di-ammonium phosphate per planting hole. Two weeks after planting, the seedlings were additionally fertilized with $6 \mathrm{~g}$ calcium ammonium nitrate per plant as recommended based on soil analyses. During the course of the experiment, the plants were irrigated manually with tap water twice a day. Plants were grown for 6 weeks. During harvest (8-12 leaf stage), fully developed leaves were collected in duplicate, each replicate consisted of about $300 \mathrm{~g}$ of fresh leaf material pooled from about 10 plants selected randomly on the field. The leaves were then freeze-dried and transported to IGZ Großbeeren, Germany, for chemical analyses.

The aerial parts of S. scabrum Mill are shown in Figure 1 below. 


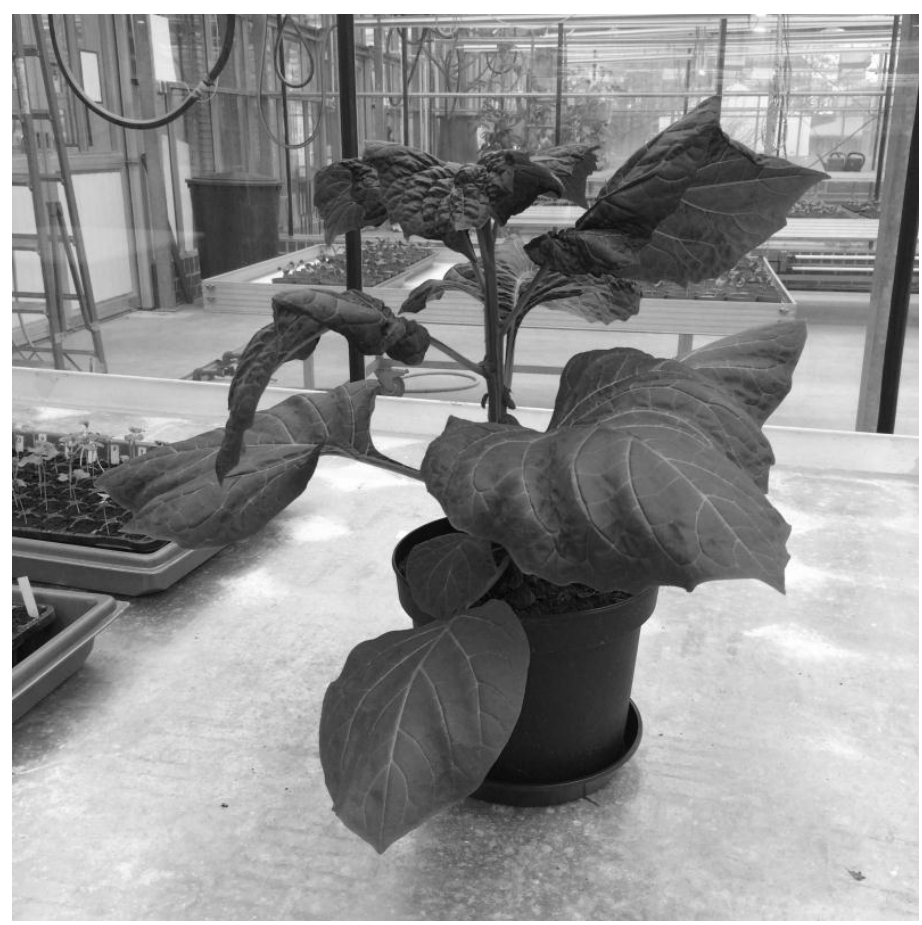

Figure 1. S. scabrum aerial parts.

Processing of S. scabrum Leaf Material

Fermentation: this was done by submerged fermentation where freshly harvested leaves; $700 \mathrm{~g}$ (location A) were washed with tap water, then immersed in $10 \mathrm{~L}$ crock pots typically used in Germany for Sauerkraut fermentation as previously described [10,11], with $2.1 \mathrm{~L}$ of a $2.5 \%$ brine solution (containing 3\% salt and 3\% sugar). The fermentation was inoculated with $1 \times 10^{7} \mathrm{CFU} / \mathrm{mL}$ of each of the starter bacteria Lactobacillus plantarum BFE 5092 and Lactobacillus fermentum BFE 6620 and left to ferment at $25{ }^{\circ} \mathrm{C}$ for $144 \mathrm{~h}$. This fermentation was followed by removal of the brine, weighing of the plant material, freeze drying and grinding to a fine powder.

Cooking (thermal treatments): this entailed boiling of the leaf material where freshly harvested leaves (location B) were washed with tap water then sliced to $1 \mathrm{~cm}$ pieces with a kitchen knife and then finally immersed in $100 \mathrm{~mL}$ of boiling water. They were cooked (simmered) for $20 \mathrm{~min}$ and then the excess water was drained out using a sieve. The drained samples were immediately cooled on ice and then frozen. They were freeze dried and ground to a fine powder.

UV-C treatment: The freshly harvested leaves of S. scabrum were immediately treated with UV-C in an UV-C chamber (ABOX ${ }^{\circledR}$ UV Technology, UMEX GmbH, Germany), where temperature and relative air humidity were kept constant at $5{ }^{\circ} \mathrm{C}$ and $85 \%$, respectively. UV-C dosage was achieved with medium pressure mercury vapour discharge lamps with a peak emission at $254 \mathrm{~nm}$ (VL-6C, 6 W-254 nm Tube, Power: $11 \mathrm{~W}$, Vilber Lourmat GmbH, Germany). The lamps were placed at a distance of $0.4 \mathrm{~m}$ to leaves. The dosage was calculated from the product of exposure time and irradiance, as measured by a portable handheld digital radiometer (UVPAD-E, Opsytec Dr. Gröbel GmbH, Germany). Based on this, two different dosages were applied, that is, $1.7 \mathrm{~kJ} \mathrm{~m}^{-2}$ and $3.4 \mathrm{~kJ} \mathrm{~m}^{-2}$. Non-treated plants served as control. Thereafter, leaves were stored at $5{ }^{\circ} \mathrm{C}$ ( $85 \%$ relative Humidity: $\left.\mathrm{RH}\right)$ for 14 days and $20^{\circ} \mathrm{C}(85 \% \mathrm{RH})$ for 6 days. The leaves were then freeze dried and ground to fine powder.

\subsection{Extract Preparation by Sonication}

The solvents used for extraction of the freeze-dried plant powder were either $70 \%$ ethanol $(\mathrm{EtOH})$ or double distilled water $\left(\mathrm{ddH}_{2} \mathrm{O}\right)$ each used at a 1:10 ratio. A sample of $0.5 \mathrm{~g}$ of the plant powder was diluted in $5 \mathrm{~mL}$ of $70 \% \mathrm{EtOH}$ or ddH2O (stock concentration: $100 \mathrm{mg} / \mathrm{mL}$ ). The mixture was 
incubated using a sonicator water bath at $50^{\circ} \mathrm{C}$ for $30 \mathrm{~min}$ as previously described [12] and was sterile filtered using a $0.22 \mu \mathrm{m}$ MillexR syringe-driven filter unit (fast flow and low binding Millipore). Then, 1:3 serial dilutions were made with $70 \% \mathrm{EtOH}$ or $\mathrm{ddH}_{2} \mathrm{O}$ from the stock solution. The cells were subsequently treated with the dilutions at a 1:100 ratio resulting in final extract concentrations starting from 333 to $1.4 \mu \mathrm{g} / \mathrm{mL}$. In each of the experiments, fresh extracts were always prepared from the freeze-dried leaf powder.

\subsection{Determination of S. scabrum Phytochemical Content}

Chemical analyses were done as previously described $[12,13]$. Phenolic compounds were analysed by high pressure liquid chromatography (HPLC) (Agilent HPLC series 1100, Agilent Technologies Sales \& Services GmbH \& Co. KG, Waldbronn, Germany) coupled to an ion trap mass spectrometer (Bruker Amazon SL, Bruker, Bremen, Germany). Quantification of phenolics was done using standards of caffeoylquinic acid [chlorogenic acid], quercetin 3-O-glucoside, kaempferol 3-O glucoside and isorhamnetin-3-O-glucoside (Carl Roth, Karlsruhe, Germany), which were used for external calibration curves. Carotenoids and chlorophylls were analysed using ultra-high-pressure liquid chromatography (UHPLC) (Agilent Technologies 1290 Infinity II) coupled with time of flight mass spectrometry (ToF-MS) (Agilent Technologies 6230 TOF LC/MS) equipped with an atmospheric pressure chemical ionization source (Agilent Technologies). External standard calibration curves of each compound were used for quantification. Compounds below the quantification limit were indicated as not detected (n.d.).

\subsection{Cell Cultures}

The cell lines used were human derived liver (HepG2) cells and a genetically modified form (ARE reporter HepG2 cells). The HepG2 cell line (ACC-180) was obtained from the German Collection of Microorganisms and Cell Cultures (DSMZ; Braunschweig, Germany) while the recombinant ARE reporter-HepG2 cell line designed to monitor the nrf2 antioxidant response pathway was obtained from BPS Bioscience, Inc., (San Diego, Califonia, USA) (60513-GVO-BPS). The cells were cultured as described [12] with few modifications; the cells were grown in DMEM medium supplemented with $15 \%$ FCS and 1\% penicillin/streptomycin solution. Additionally, $600 \mu \mathrm{g} / \mathrm{mL}$ geneticin antibiotic was used in ARE reporter HepG2 cell line. Incubation was done in a $95 \%$ humidified incubator at $37^{\circ} \mathrm{C}$ and $5 \% \mathrm{CO}_{2}$.

\subsection{Assessment of Anti-Genotoxic Activity of Leaves of S. scabrum Using the Comet Assay}

The Comet assay was used to determine anti-genotoxic activity of S. scabrum which was performed according to Lamy et al. [14], with few modifications. Pre-treatment of HepG2 cells was done with either water or ethanol extracts from S. scabrum for $24 \mathrm{~h}$, experimental control were considered. The cells were then washed and exposed to $10 \mu \mathrm{M} \mathrm{AFB} 1$ or $0.1 \%$ DMSO for another $24 \mathrm{~h}$. Comet assay was then performed and analysis done with Comet 5.5 image software (Munich, Germany) connected to a Leica fluorescence microscope (Leica DMLS; Wetzlar, Germany; excitation filter: BP 546/10 nm; barrier filter: $590 \mathrm{~nm}$ ) connected to a high sensitivity charge-coupled (CCD) camera. The percent tail DNA was used as the parameter for indicating DNA damage.

\subsection{Determination of Anti-Oxidant Activity of S. scabrum}

The anti-oxidant activity of S. scabrum was determined by electron paramagnetic resonance spectroscopy (EPR) equipped with temperature and gas controller Bio III (Noxygen, Elzach, Germany) which is capable of detecting ROS in the cells. HepG2 cells pre-treated with water or ethanolic extracts from S. scabrum were used. The EPR spectroscopy protocol previously described by Lamy et al. [15] was adopted with few modifications. HepG2 cells were exposed to (a) $200 \mu \mathrm{M}$ menadione or $0.1 \%$ DMSO (solvent control) for 30 min or (b) $100 \mu \mathrm{M}$ ferrous sulphate or supplemented DMEM medium (solvent control) for $15 \mathrm{~min}$. The cells were then washed with pre-warmed Krebs-HEPES buffer (KHB) followed by either $30 \mathrm{~min}$ incubation at $37{ }^{\circ} \mathrm{C}$ with spin probe $100 \mu \mathrm{M} \mathrm{CMH}$ in 
KHB supplemented with $25 \mu \mathrm{M}$ DFO and $5 \mu \mathrm{M}$ DETC, or 120 min incubation at $37{ }^{\circ} \mathrm{C}$ with 1-hydroxy-4-phosphono-oxy-2,2,6,6-tetramethyl-piperidinethe (PPH) at a concentration of $200 \mu \mathrm{M}$ in KHB supplemented as mentioned above. Supernatants were transferred to new reaction tubes and kept on ice. A 10 scan screening was done for each sample using $50 \mu \mathrm{L}$ glass capillaries.

\subsection{Assessing Induction of Nrf2 Antioxidant Pathway by S. scabrum}

The ARE/Nrf2 reporter gene activity was measured to determine the induction of the Nrf2 anti-oxidant pathway which was analysed according to the manufacturer's instructions using the ONE-Glo $^{\text {TM }}$ Luciferase Assay System (Promega GmbH, Mannheim, Germany). In brief, HepG2-ARE cells were seeded in 96 well plates $\left(4 \times 10^{4}\right.$ cells/well $)$ and immediately exposed to the S. scabrum extracts. After incubation for $18 \mathrm{~h}$, cells were lysed and luminescence measured $15 \mathrm{~min}$ after substrate addition using an infinite M200 microplate reader (Infinite M200, Tecan Group Ltd., Männedorf, Switzerland).

\subsection{Measurement of Cytotoxicity and Cytostatic Activity}

Cytotoxicity was determined by checking the viability of HepG2 cells treated with S. scabrum extract or solvent control after $48 \mathrm{~h}$ using the trypan blue dye exclusion test. Total numbers of extract treated cells were compared with the solvent control. Counting was done using an Neubauer improved counting chamber (Brand GmbH \& Co. KG, Wertheim, Germany).

\subsection{Data Analysis}

All data were analysed using Graph Pad Prism 6 (GraphPad Software Inc., San Diego, CA, USA). Results of the cell culture experiments are presented as means of at least three independent experiments. Differences were considered significant at $p \leq 0.05\left({ }^{*}\right), p \leq 0.01\left(^{* *}\right)$. Statistical significance was assessed using two-way analysis of variance (ANOVA) followed by Dunnett's multiple comparisons test.

\section{Results}

\subsection{Phytochemical Composition of Raw, Processed and UV-C Treated S. scabrum}

The impact of cultivation and processing condition on the content of secondary plant metabolites is presented in Tables 1-5. Phenolic compounds, carotenoids and chlorophylls were analysed in the plant extracts. The highest amounts of phenolic compounds $(19,644.4 \mu \mathrm{g} / \mathrm{mL})$ or $(196,444 \mu \mathrm{g} / \mathrm{g}$ dry matter (DM) of leaves) were detected in raw extracts from plants grown in open greenhouse from location B (see Table 4). The least was found in the cooked ethanolic extracts from location B $(3024.2 \mu \mathrm{g} / \mathrm{mL})$ or $(30,242 \mu \mathrm{g} / \mathrm{g}$ DM) (see Table 2). Carotenoids and chlorophylls were not detected or only at minor amounts in water extract (see Table 1 and 2) but in ethanolic extracts, the content of carotenoids ranged from $17 \mu \mathrm{g} / \mathrm{mL}$ or $170 \mu \mathrm{g} / \mathrm{g} \mathrm{DM}$ (raw plant extracts from open greenhouses) to $179 \mu \mathrm{g} / \mathrm{mL}$ or $1790 \mu \mathrm{g} / \mathrm{g}$ DM (fermented plant extracts). The chlorophyll content ranged from 5 $\mu \mathrm{g} / \mathrm{mL}$ or $50 \mu \mathrm{g} / \mathrm{g} \mathrm{DM}$ in the fermented plant extract to $464 \mu \mathrm{g} / \mathrm{mL}$ or $4640 \mu \mathrm{g} / \mathrm{g}$ DM in the raw plant extract from location $C$. 
Table 1. Phytochemical content in the extracts of raw vs. fermented S. scabrum in $\mu \mathrm{g} / \mathrm{g}$ DM (location A). The data are presented as phytochemical content \pm average analytical error.

\begin{tabular}{|c|c|c|c|c|}
\hline Polyphenols & Raw Ethanol & Raw Water & $\begin{array}{l}\text { Fermented } \\
\text { Ethanol }\end{array}$ & Fermented Water \\
\hline \multicolumn{5}{|l|}{ tentative structure } \\
\hline 3-caffeoylquinic acid & $1314 \pm 26$ & $828 \pm 41$ & $1186 \pm 24$ & $1493 \pm 30$ \\
\hline 5-caffeoylquinic acid & $1023 \pm 20$ & $1365 \pm 27$ & $1490 \pm 30$ & $2315 \pm 46$ \\
\hline 4-caffeoylquinic acid & $30,839 \pm 617$ & $8311 \pm 166$ & $5394 \pm 108$ & $15,032 \pm 301$ \\
\hline caffeoylmalate & n.d. & $1574 \pm 31$ & $3688 \pm 74$ & $1418 \pm 28$ \\
\hline caffeoylmalate & $52,628 \pm 1053$ & $34,750 \pm 695$ & $12,527 \pm 251$ & $35,211 \pm 704$ \\
\hline quercetin-3-glucosylrhamnogalcatoside & $185,237 \pm 3704$ & $2083 \pm 42$ & $479 \pm 24$ & $994 \pm 50$ \\
\hline coumaric acid & $1622 \pm 32$ & $653 \pm 33$ & $850 \pm 43$ & $533 \pm 27$ \\
\hline quercetin-3-rhamnogalactoside & $422 \pm 21$ & $438 \pm 22$ & n.d. & n.d. \\
\hline $\begin{array}{l}\text { quercetin-3-rhamnosylrhamnogalactoside } \\
\text { (isomer 2) }\end{array}$ & $3326 \pm 67$ & $5475 \pm 110$ & $1776 \pm 36$ & $5428 \pm 109$ \\
\hline $\begin{array}{l}\text { quercetin-3-rhamnosylrhamnogalactoside } \\
\text { (isomer 3) }\end{array}$ & $1649 \pm 33$ & $2396 \pm 48$ & $932 \pm 47$ & $2930 \pm 59$ \\
\hline sinapoylmalate & $608 \pm 30$ & $644 \pm 32$ & $566 \pm 28$ & $809 \pm 40$ \\
\hline kaempferol-3-diglucoside & $423 \pm 21$ & $643 \pm 32$ & $538 \pm 27$ & $1036 \pm 21$ \\
\hline $\begin{array}{l}\text { kaempferol-3-rhamnosylrhamnogalactoside } \\
\text { (isomer 1) }\end{array}$ & $528 \pm 26$ & $868 \pm 43$ & $564 \pm 28$ & $589 \pm 29$ \\
\hline quercetin-3-pentosylrutinoside & $1787 \pm 36$ & $2090 \pm 42$ & $1024 \pm 20$ & $2661 \pm 53$ \\
\hline sinapic acid & $1705 \pm 34$ & $539 \pm 27$ & $1131 \pm 23$ & $2742 \pm 55$ \\
\hline $\begin{array}{l}\text { kaempferol-3-rhamnosylrhamnogalactoside } \\
\text { (isomer 1) }\end{array}$ & $892 \pm 45$ & $688 \pm 34$ & $610 \pm 31$ & $1004 \pm 20$ \\
\hline quercetin-3-rutinoside & $1346 \pm 27$ & $709 \pm 35$ & $911 \pm 46$ & $1737 \pm 35$ \\
\hline Total & $101,964 \pm 2039$ & $64,054 \pm 1281$ & $33,666 \pm 673$ & $75,343 \pm 1507$ \\
\hline \multicolumn{5}{|l|}{ Carotenoids } \\
\hline$\beta$-carotene & $1 \pm 0.1$ & n.d. & $1 \pm 0.1$ & n.d. \\
\hline zeaxanthin & $70 \pm 7.0$ & $1 \pm 0.1$ & $1326 \pm 132.6$ & $4 \pm 0.4$ \\
\hline lutein & $515 \pm 51.5$ & $1 \pm 0.1$ & $462 \pm 46.2$ & n.d. \\
\hline Total & $586 \pm 58.6$ & $2 \pm 0.2$ & $1789 \pm 178.9$ & $4 \pm 0.4$ \\
\hline \multicolumn{5}{|l|}{ Chlorophylls } \\
\hline chlorophyll a & $3500 \pm 350.0$ & n.d. & n.d. & n.d. \\
\hline chlorophyll b & $1076 \pm 107.6$ & n.d. & $50 \pm 5.0$ & n.d. \\
\hline Total & $4576 \pm 457.6$ & n.d. & $50 \pm 5.0$ & n.d. \\
\hline
\end{tabular}

(n.d. $=$ not detected). Phytochemical classes are put in bold. 
Table 2. Phytochemical content in the extracts of raw vs. cooked S. scabrum in $\mu$ g/g DM (location B). The data are presented as phytochemical content \pm average analytical error.

\begin{tabular}{|c|c|c|c|c|}
\hline Polyphenols & Raw Ethanol & Raw Water & $\begin{array}{l}\text { Cooked } \\
\text { Ethanol }\end{array}$ & Cooked Water \\
\hline \multicolumn{5}{|l|}{ tentative structure } \\
\hline 3-caffeoylquinic acid & $1390 \pm 28$ & $1626 \pm 33$ & $1314 \pm 26$ & $1879 \pm 38$ \\
\hline 5-caffeoylquinic acid & $1231 \pm 25$ & $1637 \pm 33$ & $1409 \pm 28$ & $2273 \pm 45$ \\
\hline 4-caffeoylquinic acid & $4946 \pm 99$ & $4630 \pm 93$ & $2460 \pm 49$ & $4567 \pm 91$ \\
\hline caffeoylmalate & $800 \pm 40$ & $3226 \pm 65$ & $702 \pm 35$ & $2513 \pm 52$ \\
\hline caffeoylmalate & $31,178 \pm 624$ & $65,157 \pm 1303$ & $22,048 \pm 441$ & $58,559 \pm 1171$ \\
\hline quercetin-3-glucosylrhamnogalcatoside & $611 \pm 31$ & $734 \pm 37$ & $548 \pm 27$ & $611 \pm 31$ \\
\hline coumaric acid & $598 \pm 30$ & $913 \pm 46$ & $650 \pm 33$ & $811 \pm 41$ \\
\hline quercetin-3-rhamnogalactoside & $367 \pm 18$ & $247 \pm 12$ & n.d. & n.d. \\
\hline $\begin{array}{l}\text { quercetin-3-rhamnosylrhamnogalactoside } \\
\text { (isomer 2) }\end{array}$ & $901 \pm 18$ & $1198 \pm 24$ & n.d. & $856 \pm 43$ \\
\hline $\begin{array}{l}\text { quercetin-3-rhamnosylrhamnogalactoside } \\
\text { (isomer 3) }\end{array}$ & $541 \pm 27$ & $796 \pm 40$ & n.d. & $629 \pm 31$ \\
\hline sinapoylmalate & $520 \pm 26$ & $540 \pm 27$ & n.d. & n.d. \\
\hline kaempferol-3-diglucoside & n.d. & $423 \pm 21$ & n.d. & n.d. \\
\hline $\begin{array}{l}\text { kaempferol-3-rhamnosylrhamnogalactoside } \\
\text { (isomer 1) }\end{array}$ & $430 \pm 22$ & $506 \pm 25$ & $398 \pm 20$ & $445 \pm 22$ \\
\hline $\begin{array}{c}\text { quercetin-3-pentosylrutinoside } \\
\text { sinapic acid }\end{array}$ & $\begin{array}{l}319 \pm 16 \\
\text { n.d. }\end{array}$ & $\begin{array}{l}369 \pm 18 \\
\text { n.d. }\end{array}$ & $\begin{array}{l}272 \pm 14 \\
\quad \text { n.d. }\end{array}$ & $\begin{array}{l}309 \pm 15 \\
\text { n.d. }\end{array}$ \\
\hline $\begin{array}{l}\text { kaempferol-3-rhamnosylrhamnogalactoside } \\
\text { (isomer 1) }\end{array}$ & $376 \pm 8$ & $387 \pm 8$ & n.d. & n.d. \\
\hline quercetin-3-rutinoside & $518 \pm 26$ & $497 \pm 25$ & $441 \pm 22$ & $393 \pm 20$ \\
\hline Total & $44,726 \pm 895$ & $82,886 \pm 1658$ & $30,242 \pm 605$ & $73,845 \pm 1477$ \\
\hline \multicolumn{5}{|l|}{ Carotenoids } \\
\hline$\beta$-carotene & $1 \pm 0.1$ & n.d. & $1 \pm 0.1$ & $1 \pm 0.1$ \\
\hline zeaxanthin & $50 \pm 5.0$ & n.d. & $41 \pm 4.1$ & $1 \pm 0.1$ \\
\hline lutein & $640 \pm 64.0$ & n.d. & $355 \pm 35.5$ & $6 \pm 0.6$ \\
\hline Total & $691 \pm 69.1$ & n.d. & $397 \pm 39.7$ & $8 \pm 0.8$ \\
\hline \multicolumn{5}{|l|}{ Chlorophylls } \\
\hline chlorophyll a & $1991 \pm 199.1$ & n.d. & $898 \pm 89.8$ & $17 \pm 1.7$ \\
\hline chlorophyll b & $1272 \pm 127.2$ & n.d. & $583 \pm 58.3$ & n.d. \\
\hline Total & $3263 \pm 326.3$ & n.d. & $1481 \pm 148.1$ & $17 \pm 1.7$ \\
\hline
\end{tabular}

(n.d. = not detected). Phytochemical classes are put in bold. 
Table 3. Phytochemical content of extracts of untreated vs. UV-C treated S. scabrum in $\mu \mathrm{g} / \mathrm{g}$ DM (location $\mathrm{C}$ ). The data are presented as phytochemical content \pm average analytical error.

\begin{tabular}{ccc}
\hline Polyphenols & Untreated Ethanol & UV-C Treated Ethanol \\
\hline tentative structure & & \\
3-caffeoylquinic acid & $1658 \pm 33$ & $1042 \pm 21$ \\
5-caffeoylquinic acid & $4009 \pm 80$ & $4715 \pm 94$ \\
4-caffeoylquinic acid & $34,633 \pm 693$ & $38,998 \pm 780$ \\
caffeoylmalate & $820 \pm 16$ & $775 \pm 39$ \\
caffeoylmalate & $18,594 \pm 372$ & $26,400 \pm 528$ \\
coumaric acid & $1928 \pm 39$ & $2490 \pm 50$ \\
quercetin-3-glucosylrhamnogalcatoside & $1215 \pm 24$ & $784 \pm 39$ \\
quercetin-3-rhamnogalactoside & $388 \pm 19$ & $439 \pm 22$ \\
quercetin-3-rhamnosylrhamnogalactoside (isomer 2) & $2912 \pm 58$ & $3436 \pm 69$ \\
quercetin-3-rhamnosylrhamnogalactoside (isomer 3) & $1546 \pm 31$ & $1832 \pm 37$ \\
sinapoylmalate & $571 \pm 29$ & $612 \pm 31$ \\
kaempferol-3-diglucoside & $433 \pm 22$ & $475 \pm 24$ \\
(isomer 1) & $574 \pm 29$ & $594 \pm 30$ \\
kaempferol-3-rhamosylrhamnogalactoside & $1126 \pm 23$ & $1332 \pm 27$ \\
quercetin-3-pentosylrutinoside & $1431 \pm 29$ & $1329 \pm 27$ \\
sinapic acid & $651 \pm 33$ & $628 \pm 31$ \\
(isomer 1) & $1045 \pm 21$ & $1103 \pm 22$ \\
kaempferol-3-rhamosylrhamnogalactoside & $73,534 \pm 1471$ & $86,984 \pm 1740$ \\
\hline Total & & \\
\hline Carotenoids & $1 \pm 0.1$ & $111 \pm 11.1$ \\
\hline B-carotene & $74 \pm 7.4$ & $541 \pm 54.1$ \\
zeaxanthin & $557 \pm 55.7$ & $653 \pm 65.3$ \\
\hline lutein & $632 \pm 63.2$ & $1929 \pm 192.9$ \\
Total & & $2944 \pm 294.4$ \\
\hline Chlorophylls & $3250 \pm 325.0$ &
\end{tabular}

Phytochemical classes are put in bold. 
Table 4. Phytochemical content in the extracts of raw vs. fermented S. scabrum grown in Normal-greenhouse ( $\mathrm{Ng}$ ) vs. Open-greenhouse $(\mathrm{Og})$ in $\mu \mathrm{g} / \mathrm{g} \mathrm{DM}$ (location B). The data are presented as phytochemical content \pm average analytical error.

\begin{tabular}{|c|c|c|}
\hline Polyphenols & Ng Ethanol & Og Ethanol \\
\hline \multicolumn{3}{|l|}{ tentative structure } \\
\hline 3-caffeoylquinic acid & $3815 \pm 76$ & $3636 \pm 73$ \\
\hline 5-caffeoylquinic acid & $6669 \pm 133$ & $15,526 \pm 311$ \\
\hline 4-caffeoylquinic acid & $28,790 \pm 576$ & $114,700 \pm 2294$ \\
\hline caffeoylmalate & $773 \pm 15$ & n.d. \\
\hline caffeoylmalate & $31,704 \pm 634$ & $42,237 \pm 845$ \\
\hline quercetin-3-glucosylrhamnogalcatoside & $2035 \pm 41$ & $3096 \pm 62$ \\
\hline coumaric acid & $1501 \pm 30$ & $1603 \pm 32$ \\
\hline quercetin-3-rhamnogalactoside & $377 \pm 19$ & $627 \pm 31$ \\
\hline quercetin-3-rhamnosylrhamnogalactoside (isomer 2) & $3705 \pm 74$ & $4464 \pm 89$ \\
\hline quercetin-3-rhamnosylrhamnogalactoside (isomer 3) & $1620 \pm 32$ & $1555 \pm 31$ \\
\hline sinapoylmalate & $522 \pm 26$ & $550 \pm 28$ \\
\hline kaempferol-3-diglucoside & $457 \pm 9$ & $541 \pm 11$ \\
\hline $\begin{array}{l}\text { kaempferol-3-rhamnosylrhamnogalactoside } \\
\text { (isomer 1) }\end{array}$ & $611 \pm 31$ & $550 \pm 28$ \\
\hline quercetin-3-pentosylrutinoside & $1037 \pm 52$ & $788 \pm 39$ \\
\hline sinapic acid & $1113 \pm 22$ & $1106 \pm 22$ \\
\hline $\begin{array}{l}\text { kaempferol-3-rhamnosylrhamnogalactoside } \\
\text { (isomer 1) }\end{array}$ & $968 \pm 19$ & $1337 \pm 27$ \\
\hline quercetin-3-rutinoside & $2393 \pm 48$ & $4128 \pm 83$ \\
\hline Total & $88,090 \pm 1762$ & $196,444 \pm 3929$ \\
\hline \multicolumn{3}{|l|}{ Carotenoids } \\
\hline$\beta$-carotene & $1 \pm 0.1$ & $1 \pm 0.1$ \\
\hline zeaxanthin & $24 \pm 2.4$ & $65 \pm 6.5$ \\
\hline lutein & $187 \pm 18.7$ & $104 \pm 10.4$ \\
\hline Total & $212 \pm 21.2$ & $170 \pm 17.0$ \\
\hline \multicolumn{3}{|l|}{ Chlorophylls } \\
\hline chlorophyll a & $2870 \pm 287.0$ & $1884 \pm 188.4$ \\
\hline chlorophyll b & $856 \pm 85.6$ & $539 \pm 53.9$ \\
\hline Total & $3726 \pm 372.6$ & $2424 \pm 242.4$ \\
\hline
\end{tabular}

(n.d. = not detected). Phytochemical classes are put in bold. 
Table 5. Phytochemical content in the extracts of $S$. scabrum in $\mu \mathrm{g} / \mathrm{g}$ DM (location D). The data are presented as phytochemical content \pm average analytical error.

\begin{tabular}{|c|c|}
\hline Polyphenols & Raw_K Ethanol \\
\hline \multicolumn{2}{|l|}{ tentative structure } \\
\hline 3-caffeoylquinic acid & $1899 \pm 38$ \\
\hline 5-caffeoylquinic acid & $1064 \pm 21$ \\
\hline 4-caffeoylquinic acid & $7826 \pm 157$ \\
\hline caffeoylmalate & $6163 \pm 123$ \\
\hline caffeoylmalate & $33,424 \pm 668$ \\
\hline quercetin-3-glucosylrhamnogalcatoside & $3007 \pm 60$ \\
\hline coumaric acid & $2118 \pm 42$ \\
\hline quercetin-3-rhamnogalactoside & $639 \pm 32$ \\
\hline quercetin-3-rhamnosylrhamnogalactoside (isomer 2) & $5749 \pm 115$ \\
\hline quercetin-3-rhamnosylrhamnogalactoside (isomer 3) & $2722 \pm 54$ \\
\hline sinapoylmalate & n.d. \\
\hline kaempferol-3-diglucoside & $514 \pm 26$ \\
\hline kaempferol-3-rhamnosylrhamnogalactoside (isomer 1) & $821 \pm 41$ \\
\hline quercetin-3-pentosylrutinoside & $1177 \pm 24$ \\
\hline sinapic acid & $1049 \pm 21$ \\
\hline kaempferol-3-rhamnosylrhamnogalactoside (isomer 1) & $1972 \pm 39$ \\
\hline quercetin-3-rutinoside & $8318 \pm 166$ \\
\hline Total & $78,462 \pm 1569$ \\
\hline \multicolumn{2}{|l|}{ Carotenoids } \\
\hline$\beta$-carotene & $1 \pm 0.1$ \\
\hline zeaxanthin & $51 \pm 5.1$ \\
\hline lutein & $141 \pm 14.1$ \\
\hline Total & $193 \pm 19.3$ \\
\hline \multicolumn{2}{|l|}{ Chlorophylls } \\
\hline chlorophyll a & $2152 \pm 215.2$ \\
\hline chlorophyll b & $672 \pm 67.2$ \\
\hline Total & $2824 \pm 282.4$ \\
\hline
\end{tabular}

(n.d. = not detected). K means grown in Kenya. Phytochemical classes are put in bold.

\subsection{Effect of Cultivation and Processing on the Protective Potential of $S$. scabrum against AFB Induced DNA Damage}

The effect of S. scabrum extracts against aflatoxin induced DNA damage was assessed using the comet assay. Basically, all the extracts tested (different cultivation environment and processing conditions) were protective against $\mathrm{AFB}_{1}$ induced damage by more than $30 \%$ at the highest concentration tested $(111 \mu \mathrm{g} / \mathrm{mL})$ as shown in Figures 2 and 3. The anti-genotoxic potential against $\mathrm{AFB}_{1}$ of raw extracts from plants cultivated within locations in Germany varied between 34 to $51 \%$ (Germany, location A: 34\%, location B; 45\%, location C: 51\%) or Kenya (48\%). Cultivation under open or normal greenhouse conditions did not significantly affect the anti-genotoxic potential (open greenhouse: 53\% vs. normal greenhouse: $47 \%$ ), Figure 3. Overall, processing in terms of fermentation or cooking further increased the anti-genotoxic potential of the plant extract as compared to the raw material (fermented: $60 \%$ vs. raw: 34\%; cooked: $49 \%$ vs. raw: $45 \%$ ). With $60 \%$ inhibition, the fermented ethanolic extract demonstrated the strongest protection against $\mathrm{AFB}_{1}$ (see Figure $2 \mathrm{~A}$ ).

When plants were treated with UV-C after harvesting, the anti-genotoxic potential was less at $37 \mu \mathrm{g} / \mathrm{mL}$ as compared to the untreated plant material but this was compensated for at the highest concentration tested (UV-C treated: $53 \%$ vs. control 51\%), Figure 3. The results derived by water extraction were comparable to ethanol extraction (Figure 2). 


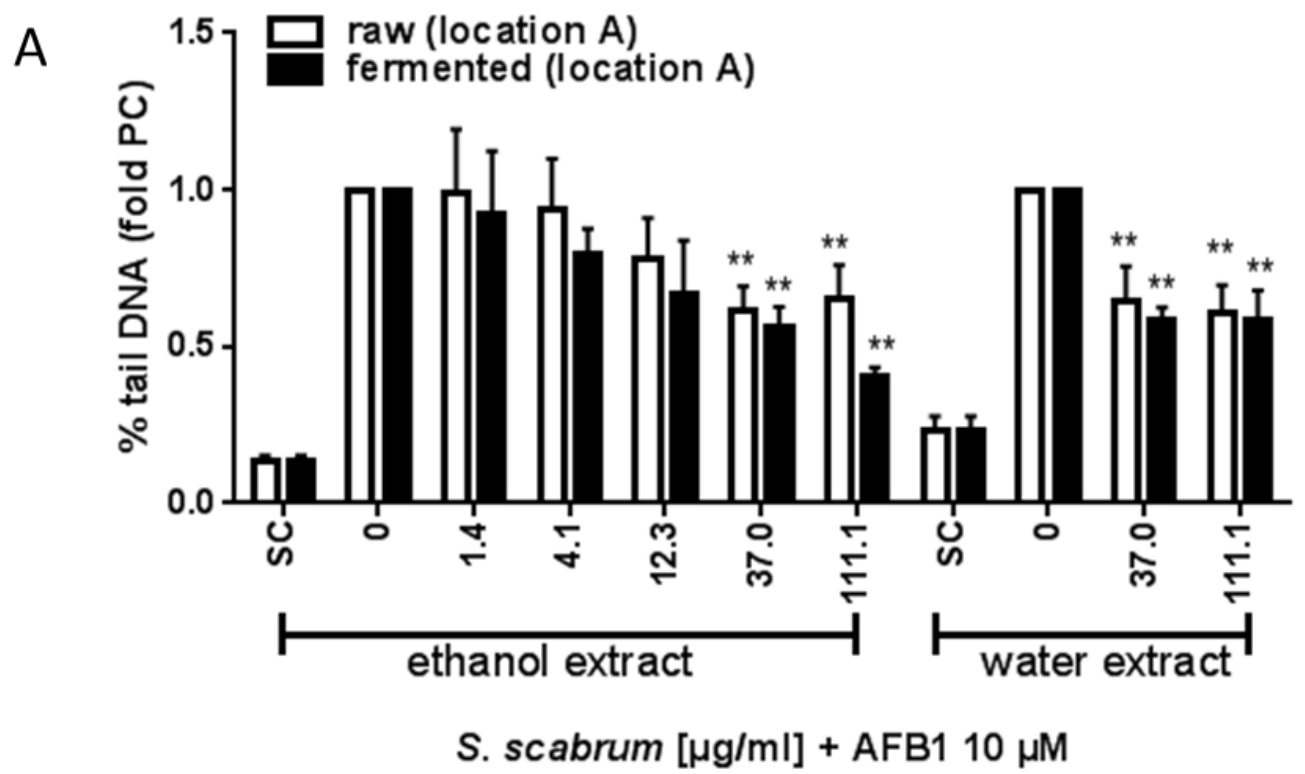

B

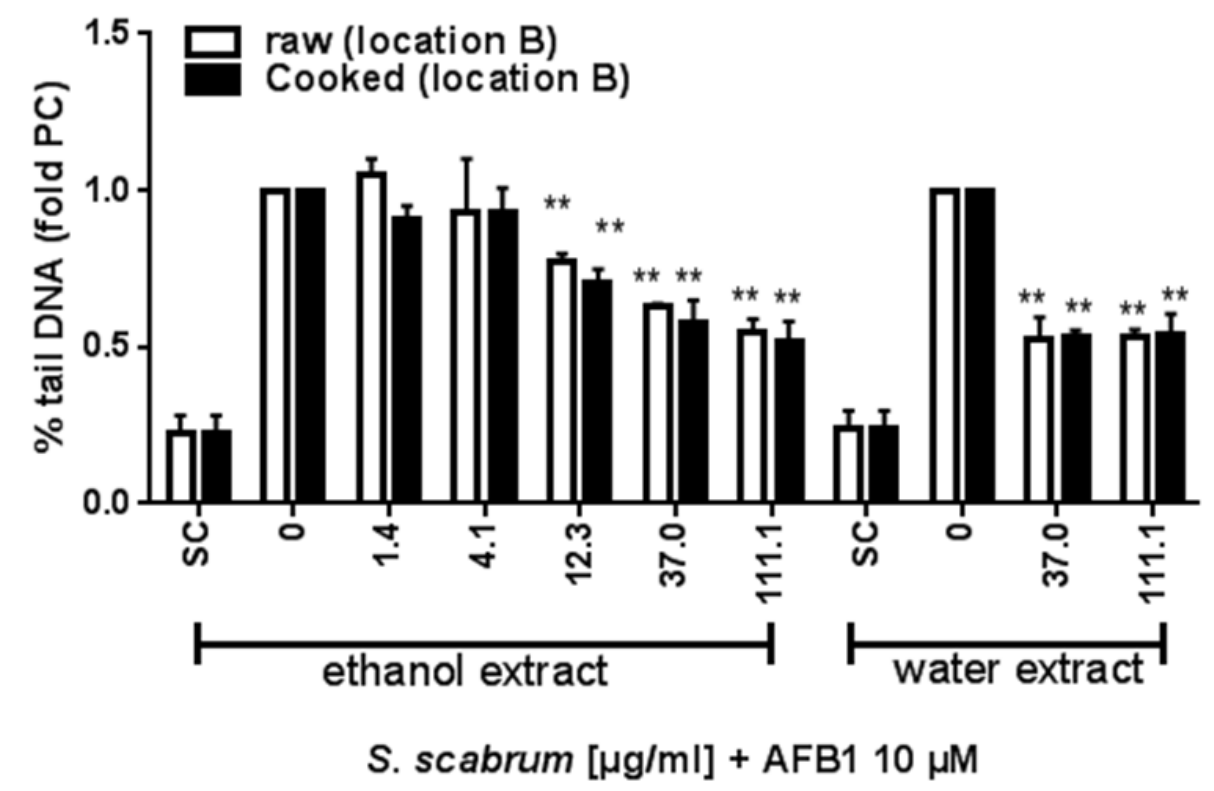

Figure 2. Anti-genotoxic activity of raw and processed S. scabrum. Results derived from the Comet assay are shown as percent tail DNA $(\mathbf{A}, \mathbf{B})$ calculated relative to $\mathrm{AFB}_{1}$-treated cells. SC: solvent control, $0.1 \%$ DMSO $+0.7 \%$ ethanol (for ethanolic extracts) or $0.1 \%$ DMSO $+\mathrm{ddH}_{2} \mathrm{O}$ (for water extracts). Data are means \pm SEM of three independent experiments. Asterisks indicate statistically significant differences between the respective treatment and the positive control (without $S$. scabrum leaf extract) $\left.p \leq 0.01{ }^{* *}\right)$. 


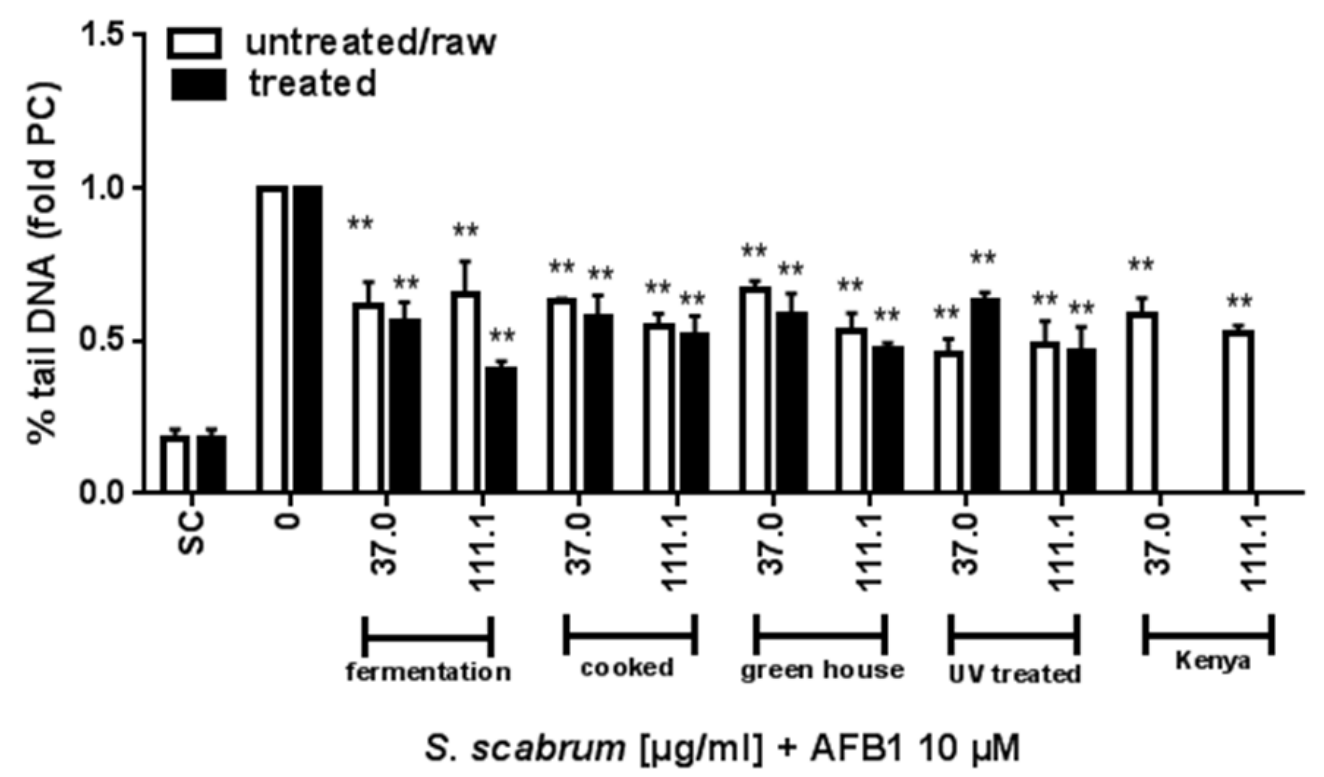

Figure 3. Impact of cultivation, processing and UV-C on the anti-genotoxic activity of S. scabrum against $\mathrm{AFB}_{1}$. Results derived from the Comet assay are shown as percent tail DNA calculated relative to $\mathrm{AFB}_{1}$-treated cells. SC: solvent control, $0.1 \%$ DMSO $+0.7 \%$ ethanol. Data are means $\pm \mathrm{SEM}$ of three independent experiments. Asterisks indicate statistically significant differences between the respective treatment and the positive control (without $S$. scabrum leaf extract) $p \leq 0.01\left(^{* *}\right)$.

\subsection{ROS Scavenging Activity and Induction of ARE/Nrf2-Mediated Gene Expression}

First, the capacity of ROS scavenging by the plant extracts was investigated using EPR. ROS production by menadione or $\mathrm{FeSO}_{4}$ resulted in a 4-fold or 2.2-fold increase, respectively over the solvent control (data not shown). In both experimental settings either using menadione or $\mathrm{FeSO}_{4}$ as stressor, all the tested extracts showed the potential to reduce stimulated ROS production (Figure 4 $\mathrm{A}-\mathrm{C})$. At the highest concentration tested, reduction in ROS production triggered by menadione ranged between $26 \%$ by plant extracts from location A (Figure $4 \mathrm{~A}$ ) to $24 \%$ from location B (Figure $4 \mathrm{~B}$ ). Processing either by fermentation or cooking did not impact the antioxidant activity as compared to the raw extract (fermented extract: $31 \%$ reduction, cooked extract: $28 \%$ reduction). A similar observation was made for ROS inhibition triggered by $\mathrm{FeSO}_{4}$ (Figure $4 \mathrm{C}$ ) although the inhibitory potency was somewhat higher compared to that seen with ROS inhibition induced by menadione. Inhibition was $38 \%$ by the fermented extract from location A, $26 \%$ by the cooked extract from location B and $38 \%$ by the UV-C treated extract from location C (Figure 4C).

Additionally, the potential of the extracts to activate the Nrf2 antioxidant pathway was investigated using a Nrf2/ARE Luciferase Reporter HepG2 cell line (Figure 5A-C). In general, the effect of the extracts in this assay was very weak. Only after 100 times concentration of the plant extract, gene expression could be seen in this assay. Then, a concentration-dependent increase in ARE/Nrf2mediated gene expression was observed upon exposure to the raw extract from location A, with an up to 4 -fold induction (Figure 5A) but not after processing in terms of fermentation. Further, extracts prepared from raw and cooked plant material from location B (Figure 5B) showed a 6-fold and 8-fold increase at the highest concentration tested, respectively. In contrast to this, plant material from location C, either untreated or processed had no effect in the assay (Figure 5A,C). 

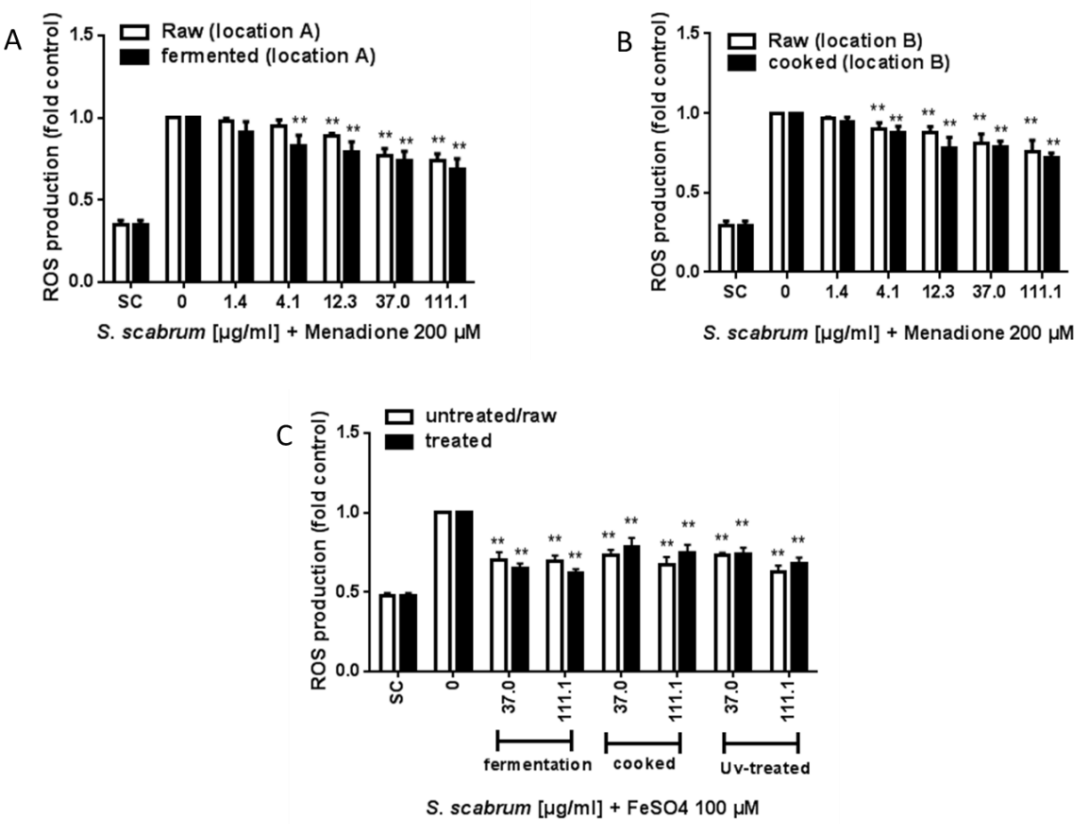

Figure 4. Anti-oxidant activity of raw, processed and UV-C treated ethanolic S. scabrum extracts. Inhibition of ROS production was determined by the EPR method in response to $200 \mu \mathrm{M}$ menadione $(\mathbf{A}, \mathbf{B})$ or $100 \mu \mathrm{M} \mathrm{FeSO}_{4}(\mathbf{C})$ in HepG2 cells. Data are means \pm SEM of three independent experiments expressed as fold control (SC: solvent control, $0.1 \%$ DMSO + 0.7\% ethanol (A,B) or supplemented DMEM medium $(\mathbf{C}))+0.7 \%$ ethanol). Asterisks indicate statistically significant differences between the respective treatment and the positive control (without $S$. scabrum leaf extract) $p \leq 0.01\left({ }^{* *}\right.$ ).
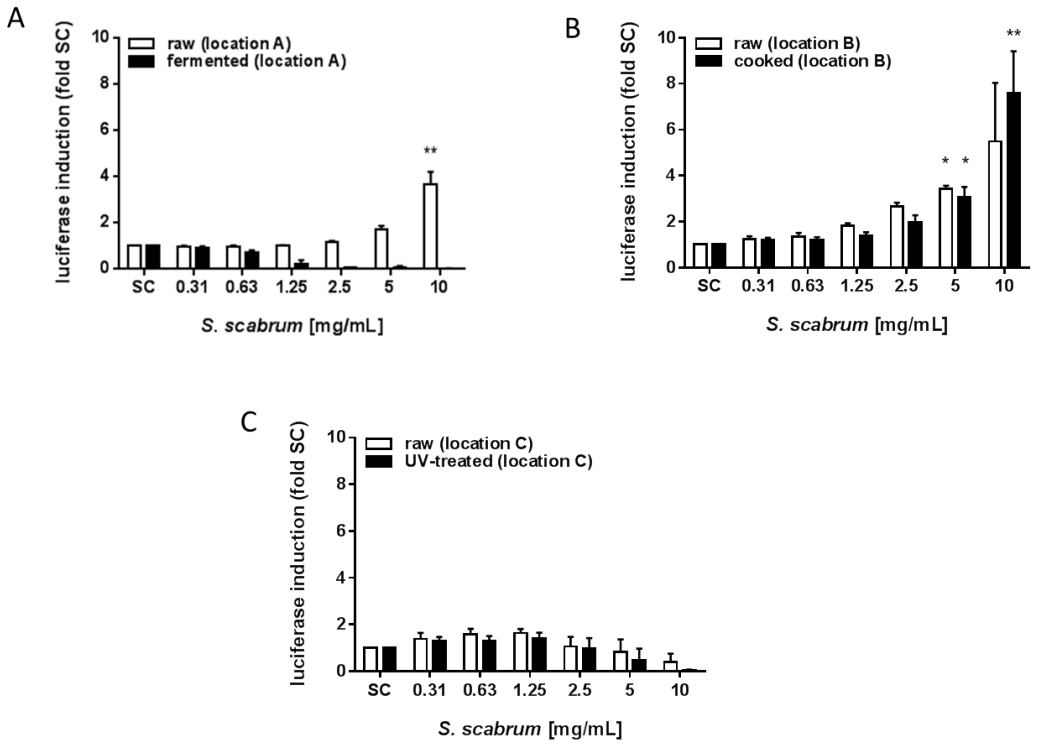

Figure 5. Induction of ARE/Nrf2- mediated gene expression by ethanolic extracts of $S$. scabrum (raw, processed and UV-C treated). Results are fold induction of luciferase as indicator of ARE/Nrf-2-mediated gene expression. Luciferase induction is given for (A) raw vs. fermented, (B) raw vs. cooked and (C) raw vs. UV-treated S. scabrum leaves. Data are means \pm SEM of three independent experiments expressed as fold control (SC: solvent control, $0.7 \%$ ethanol). Asterisks indicate statistically significant differences between the respective treatment and the solvent control $\left.p \leq 0.05\left(^{*}\right) p \leq 0.01{ }^{* *}\right)$. 


\subsection{Induction of Cytotoxicity by Ethanolic S. scabrum Extracts}

Relevant cytotoxicity could not be observed upon treatment of the cells (HepG2) with the extracts of S. scabrum for $48 \mathrm{~h}$ as shown in Figure 6A-C. All the concentrations of the extract tested showed a viability $>70 \%$.

A

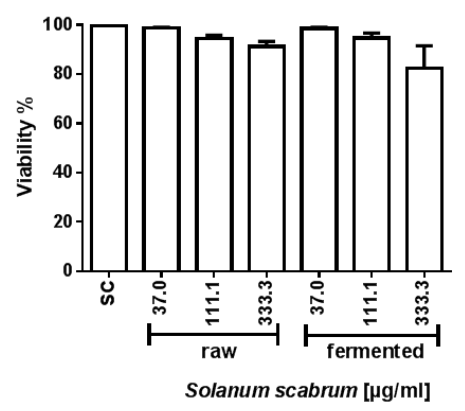

B

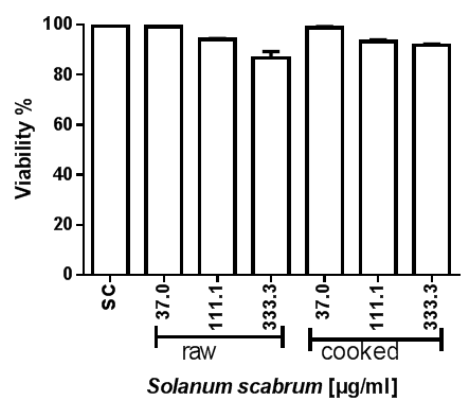

C

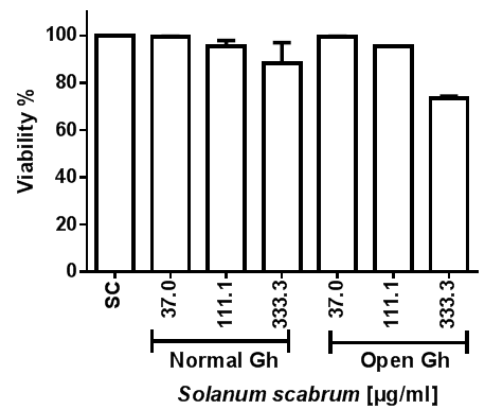

Figure 6. Cytotoxicity of ethanolic extracts of S. scabrum. HepG2 cells were treated for $48 \mathrm{~h}$ with the leaf extracts and cytotoxicity determined using trypan blue dye exclusion test. Results are given for (A) raw vs. fermented, (B) raw vs. cooked and (C) normal greenhouse vs. open green house experiments. Data are means \pm SEM of three independent experiments ( $S C=$ solvent control, $0.7 \%$ ethanol).

\section{Discussion}

The cancer preventive potential in terms of protection from DNA damage and oxidative stress of Solanum species has previously been studied but not much information has been available for the leaves. Anti-mutagenicity as determined by the micronucleus test in mice bone marrow was shown by ethanolic fruit extracts from S. lycocarpum [16] and ethanolic leaf extracts from S. paniculatum L. [17]. Aqueous leaf extract of S. nigrum have been shown to suppress mitochondrial function and epithelial mesenchymal transition in MCF-7 breast cancer cells [18] and to cause $>50 \%$ cytotoxicity in human A-375 melanoma cells [19]. The present in vitro findings suggest that basically, at non-toxic concentrations, the leaf extract of $S$. scabrum has chemopreventive properties in terms of anti-genotoxicity against $\mathrm{AFB}_{1}$ and antioxidant potential. The cultivation conditions of the plant obviously had an impact on the observed anti-genotoxic potential against $\mathrm{AFB}_{1}$ but with an observed variance of $17 \%$ it was not that marked between the raw extracts derived from different locations. This information is important because it shows that no matter if the plants were grown under open field conditions in the climate region of Germany or Kenya or under very controlled conditions such as greenhouse usage, a basic health promoting potential of the plant leaves seems to be given. Currently, there exist no standard procedure for cultivation and processing of African indigenous vegetables (AIVs). However, it is an area that is slowly drawing interest with the focus of trying to come up with the optimal cultivation conditions and processing method. So far, pre- and post-harvest procedures have been reported to impact the content of phytochemicals of the AIVs and could consequently also affect the bioactivity. As an example, the leaf extract from the medicinal plant Achillea collina grown in high altitude had a higher polyphenol level than the one grown in low altitude [20]. This was also shown for the soil water content [21]. For plant fermentation, it has been shown to impact the polyphenolic content and then also the antioxidant activity [22,23]. Thus, we addressed this issue for S. scabrum in the present study.

Now, when comparing the results on anti-genotoxicity with the data from chemical analysis, a few facts are remarkable. First, the least potent raw extract (location A) showed a reduction in $\mathrm{AFB}_{1}$-induced genotoxicity by $34 \%$, the most potent one (location C) by $51 \%$. However, the total polyphenol content of the latter one was about $30 \%$ less as compared to the one from location $\mathrm{A}$, carotenoids and chlorophylls were present in both extracts at the same range. Second, there was not 
much difference between the bioactivity of the raw and processed extracts in terms of cooking or fermentation. The anti-genotoxic potential of raw and fermented plant extracts was comparable or rather increased by fermentation. However, they differed in their total polyphenol content by about $70 \%$, whereas the fermented extract contained the minor amount of both. The two extracts did also not contain any or only low amounts of carotenoids and chlorophylls. Third, raw and cooked plant extracts were equally potent in the anti-genotoxicity assay but then, the raw plant extract contained only $50 \%$ of the total polyphenols compared to the cooked plant extract. Fourth, water and ethanolic extracts showed a comparable anti-genotoxicity even though their phytochemical content differed strongly in all analysed groups.

Polyphenols are well known for their anti-genotoxic, antioxidant and anti-cancer capacities [24-26]. These phytochemicals also can activate the NRF2 pathway and in the end result in production of antioxidant enzymes capable of reducing oxidative stress in the cells [27-29]. The analysed carotenoids contained majorly lutein and zeaxanthin which have previously been shown to have antioxidant activity [29-32] and also chlorophylls are known anti-genotoxicants and antioxidants [33]. Carotenoids have also been reported to interact synergistically with polyphenols to confer antioxidant activity [34]. In the present study, all tested extracts showed antioxidant capacity against both menadione and $\mathrm{FeSO}_{4}$ with a comparable potential. While menadione induces ROS by redox cycling via one-electron reductive enzymes [35], the iron (II) ion from $\mathrm{FeSO}_{4}$ acts as key generator in the intracellular formation of ROS such as $\mathrm{H}_{2} \mathrm{O}_{2}$ and $\mathrm{OH}$ radicals [36,37]. In another study by our group we could detect significant antioxidant activity of the AIV Amaranthus cruentus against $\mathrm{FeSO}_{4}$ but not menadione [38]. Due to the differential finding, we then argued that indeed the detected polyphenols in the plants could account for the observed anti-oxidant effect since their antioxidant activity largely depends on their iron-chelating properties [39]. This similarly could explain the activity seen here with S. scabrum against $\mathrm{FeSO}_{4}$.

Taken together, for the present study, no clear phytochemical pattern was identified, which could sufficiently explain the observations in bioactivity. For the AIV B. carinata, we already found that plant processing in terms of cooking or fermentation had an impact on the phytochemical content but this did not adversely affect the anti-genotoxicity of the plant, too [12]. Cooking then rather increased the antioxidant capacity of the ethanolic plant extract. Of course, synergistic effects of the diverse bioactive compounds as well as activity of de-glucosylated and decomposition products could also have some impact. As previously studied [40,41], the radical scavenging activity of polyphenols was reported to be stable although the original compound mixture was decomposed. In another study, synergy between compounds was proposed to be up to $24 \%$ of the observed antioxidant activity which could be explained by the calculated total phenolic compounds from red wine [42]. Besides polyphenols, carotenoids and chlorophylls [3,43], Solanum species were reported to possess a number of further potent bioactive phytochemicals such as solasodine, solanidine and solamargine that also have anticancer and antioxidant activity [44-46] but these have not been analysed here.

Photosynthetically ineffective light such as UV light has increasingly gained interest for improving postharvest quality [9], at present primarily UV-C $(190-280 \mathrm{~nm})$ applied for effective surface decontamination [47,48], directly damaging microbial DNA. However, it also affects textural properties [49] and induces the biosynthesis of plant secondary metabolites $[9,50,51]$. Thus, the present investigations also analysed whether this postharvest method might be recommended also in terms of bioactivity preservation. Present results confirmed that neither the anti-genotoxic nor antioxidant potential of the plant is adversely affected. Thus, UV-C treatment might be recommended when envisaging postharvest disinfection purposes, that is, food safety issues of AIVs in postharvest and guaranteeing no negative impact on health promoting compounds.

The present study was carried out using a human liver-derived cell line which is known for their metabolically competence in phase I and II enzyme activities [52,53]. Thus, it is a useful in vitro system to partly reflect the human liver metabolism of the phytochemicals and also the applied carcinogen $\mathrm{AFB}_{1}$. However, of course it cannot reflect the complex processes that occur during digestion of the 
plant material in the human body. Especially the gut microbiota is known to extensively metabolize phytochemicals which in consequence greatly influences their bioavailability [54]. This is certainly a limitation of the study.

\section{Conclusions}

Irrespective of the processing method, consumption of the leaves from S. scabrum might help in cancer prevention strategies, based on the present in vitro findings. Processing clearly affected the content of phytochemicals but this did not result in a diminished chemopreventive effect. However, other endpoints relevant for cancer prevention may perhaps be influenced by this change in phytochemicals, in a negative or positive way. Cooking and fermentation are simple preservation methods in terms of preventing the growth of bacteria, fungi and other microorganisms, which gives them a clear advantage to consumption of the raw plant material. Selection of the Solanum species for consumption should be carefully considered since species like $S$. nigrum have been reported to have toxic substances in certain part of the plants. The cheap UV-C postharvest treatment could be a potential alternative based on the present findings.

Author Contributions: E.L. conceived the study. E.L. and M.S. initiated the overall idea. G.A.O., N.S., S.N. and S.B. conducted the experiments and analysed the data. S.H.-K., B.N. and B.T., cultivated, harvested and processed the plant material. G.A.O. and E.L. wrote the manuscript. All authors critically revised the manuscript. All authors approved the final version of the manuscript.

Funding: This study was funded by the German Federal Ministry of Education and Research (BMBF), under the African-German project "Horticultural Innovation and Learning for Improved Nutrition and Livelihood in East Africa (HORTINLEA)" in the context of the funding measure GlobE-Global Food Security" as part of the "National Research Strategy BioEconomy 2030" framework program (project number 031A248H, 031A248J). The article processing charge was funded by the German Research Foundation (DFG) and the University of Freiburg in the funding program Open Access Publishing.

Conflicts of Interest: The authors declare no conflict of interest.

\section{References}

1. Manoko, M.L.K.; van den Berg, R.G.; Feron, R.M.C.; van der Weerden, G.M.; Mariani, C. Genetic diversity of the African hexaploid species Solanum scabrum Mill. and Solanum nigrum L. (Solanaceae). Genet. Resour. Crop. Evol. 2008, 55, 409-418. [CrossRef]

2. Jiménez-Aguilar, D.M.; Grusak, M.A. Evaluation of minerals, phytochemical compounds and antioxidant activity of Mexican, Central American, and African green leafy vegetables. Plant Foods Hum. Nutr. 2015, 70, 357-364. [CrossRef] [PubMed]

3. Neugart, S.; Baldermann, S.; Ngwene, B.; Wesonga, J.; Schreiner, M. Indigenous leafy vegetables of Eastern Africa-A source of extraordinary secondary plant metabolites. Food Res. Int. 2017, 100, 411-422. [CrossRef] [PubMed]

4. Yuan, B.; Byrnes, D.; Giurleo, D.; Villani, T.; Simon, J.E.; Wu, Q. Rapid screening of toxic glycoalkaloids and micronutrients in edible nightshades (Solanum spp.). JFDA 2018, 26, 751-760. [CrossRef] [PubMed]

5. Laher, F.; Aremu, A.O.; Van Staden, J.; Finnie, J.F. Evaluating the effect of storage on the biological activity and chemical composition of three South African medicinal plants. S. Afr. J. Bot. 2013, 88, 414-418. [CrossRef]

6. Michalska, A.; Łysiak, G. Bioactive Compounds of blueberries: Post-harvest factors influencing the nutritional value of products. IJMS 2015, 16, 18642-18663. [CrossRef] [PubMed]

7. Lu, Y.; Luthria, D. Instrumental Methods for the Analysis and Identification of Bioactive Molecules, 3th ed.; American Chemical Society: Beltsville, MD, USA, 2014; pp. 3-31.

8. Chao, J.; Dai, Y.; Cheng, H.Y.; Lam, W.; Cheng, Y.C.; Li, K.; Peng, W.H.; Pao, L.H.; Hsieh, M.T.; Qin, X.M. Improving the concentrations of the active components in the herbal tea ingredient, uraria crinita: The effect of post-harvest oven-drying processing. Sci. Rep. 2017, 7, 38763. [CrossRef] [PubMed]

9. Gogo, E.O.; Förster, N.; Dannehl, D.; Frommherz, L.; Trierweiler, B.; Opiyo, A.M.; Ulrichs, C.; Huyskens-Keil, S. Postharvest UV-C application to improve health promoting secondary plant compound pattern in vegetable amaranth. Innov. Food Sci. Emerg. Technol. 2018, 45, 426-437. [CrossRef] 
10. Oguntoyinbo, F.A.; Cho, G.S.; Trierweiler, B.; Kabisch, J.; Rösch, N.; Neve, H.; Bockelmann, W.; Frommherz, L.; Nielsen, D.S.; Krych, L.; et al. Fermentation of African kale (Brassica carinata) using L. plantarum BFE 5092 and L. fermentum BFE 6620 starter strains. Int. J. Food Microbiol. 2016, 238, 103-112. [CrossRef] [PubMed]

11. Oguntoyinbo, F.A.; Fusco, V.; Cho, G.S.; Kabisch, J.; Neve, H.; Bockelmann, W.; Huch, M.; Frommherz, L.; Trierweiler, B.; Becker, B.; et al. Produce from Africa's gardens, potential for leafy vegetable and fruit fermentations. Front Microbiol. 2016, 7, 981. [CrossRef] [PubMed]

12. Odongo, G.A.; Schlotz, N.; Herz, C.; Hanschen, F.S.; Baldermann, S.; Neugart, S.; Trierweiler, B.; Frommherz, L.; Franz, C.M.; Ngwene, B.; et al. The role of plant processing for the cancer preventive potential of Ethiopian kale (Brassica carinata). Food Nutr. Res. 2017, 61, 1271527. [CrossRef] [PubMed]

13. Schroter, D.; Baldermann, S.; Schreiner, M.; Witzel, K.; Maul, R.; Rohn, S.; Neugart, S. Natural diversity of hydroxycinnamic acid derivatives, flavonoid glycosides, carotenoids and chlorophylls in leaves of six different amaranth species. Food Chem. 2018, 267, 376-386. [CrossRef] [PubMed]

14. Lamy, E.; Kassie, F.; Gminski, R.; Schmeiser, H.H.; Mersch-Sundermann, V. 3-Nitrobenzanthrone (3-NBA) induced micronucleus formation and DNA damage in human hepatoma (HepG2) cells. Toxicol. Lett. 2004, 146, 103-109. [CrossRef] [PubMed]

15. Lamy, E.; Herz, C.; Lutz-Bonengel, S.; Hertrampf, A.; Marton, M.R.; Mersch-Sundermann, V. The MAPK pathway signals telomerase modulation in response to isothiocyanate-induced DNA damage of human liver cancer cells. PLoS ONE 2013, 8, e53240. [CrossRef] [PubMed]

16. Vieira, P.M.; Costa, P.M.; Ribeiro e Silva, C.; Chen-Chen, L. Assessment of the genotoxic, antigenotoxic, and cytotoxic activities of the ethanolic fruit extract of Solanum lycocarpum A. St. Hill. (Solanaceae) by micronucleus test in mice. J. Med. Food 2010, 13, 1409-1414. [CrossRef] [PubMed]

17. Vieira, P.M.; Paula, J.R.; Chen-Chen, L. Solanum paniculatum L. leaf and fruit extracts: Assessment of modulation of cytotoxicity and genotoxicity by micronucleus test in mice. J. Med. Food 2010, 13, 1424-1430. [CrossRef] [PubMed]

18. Lai, Y.J.; Tai, C.J.; Wang, C.W.; Choong, C.Y.; Lee, B.H.; Shi, Y.C.; Tai, C.J. Anti-cancer activity of solanum nigrum (AESN) through suppression of mitochondrial function and epithelial-mesenchymal transition (EMT) in breast cancer cells. Molecules 2016, 21, 553. [CrossRef] [PubMed]

19. Ling, B.; Michel, D.; Sakharkar, M.K.; Yang, J. Evaluating the cytotoxic effects of the water extracts of four anticancer herbs against human malignant melanoma cells. Drug Des. Dev. Ther. 2016, 10, 3563-3572. [CrossRef] [PubMed]

20. Giorgi, A.; Madeo, M.; Speranza, G.; Cocucci, M. Influence of environmental factors on composition of phenolic antioxidants of Achillea collina Becker ex Rchb. Nat. Prod. Res. 2010, 24, 1546-1559. [CrossRef] [PubMed]

21. Tatar, Ö.; Konakchiev, A.; Tsonev, T.; Velikova, V.; Gesheva, E.; Bayram, E.; Vitkova, A.; Edreva, A. Plant-soil water status-induced changes in physiological and biochemical properties of yarrow. J. Essent. Oil Bear. 2016, 19, 1776-1787. [CrossRef]

22. Rocchetti, G.; Chiodelli, G.; Giuberti, G.; Ghisoni, S.; Baccolo, G.; Blasi, F.; Montesano, D.; Trevisan, M.; Lucini, L. UHPLC-ESI-QTOF-MS profile of polyphenols in Goji berries (Lycium barbarum L.) and its dynamics during in vitro gastrointestinal digestion and fermentation. J. Funct. Foods 2018, 40, 564-572. [CrossRef]

23. Corona, G.; Coman, M.M.; Guo, Y.; Hotchkiss, S.; Gill, C.; Yaqoob, P.; Spencer, J.P.E.; Rowland, I. Effect of simulated gastrointestinal digestion and fermentation on polyphenolic content and bioactivity of brown seaweed phlorotannin-rich extracts. Mol. Nutr. Food Res. 2017, 61. [CrossRef] [PubMed]

24. Santhakumar, A.B.; Bulmer, A.C.; Singh, I. A review of the mechanisms and effectiveness of dietary polyphenols in reducing oxidative stress and thrombotic risk. J. Hum. Nutr. Diet 2014, 27, 1-21. [CrossRef] [PubMed]

25. Perron, N.R.; Brumaghim, J.L. A review of the antioxidant mechanisms of polyphenol compounds related to iron binding. Cell Biochem. Biophys. 2009, 53, 75-100. [CrossRef] [PubMed]

26. Kiemlian Kwee, J. Yin and Yang of polyphenols in cancer prevention: A short review. Anticancer Agents Med. Chem. 2016, 16, 832-840. [CrossRef] [PubMed]

27. Eggler, A.L.; Savinov, S.N. Chemical and biological mechanisms of phytochemical activation of Nrf2 and importance in disease prevention. Recent Adv. Phytochem. 2013, 43, 121-155. [PubMed]

28. Jadeja, R.N.; Upadhyay, K.K.; Devkar, R.V.; Khurana, S. Naturally occurring nrf2 activators: Potential in treatment of liver injury. Oxid. Med. Cell Longev. 2016. [CrossRef] [PubMed] 
29. Kaulmann, A.; Bohn, T. Carotenoids, inflammation, and oxidative stress-implications of cellular signaling pathways and relation to chronic disease prevention. Nutr. Res. 2014, 34, 907-929. [CrossRef] [PubMed]

30. Li, S.; Ding, Y.; Niu, Q.; Xu, S.Z.; Pang, L.J.; Ma, R.L.; Jing, M.X.; Feng, G.L.; Tang, J.X.; Zhang, Q.; et al. Lutein has a protective effect on hepatotoxicity induced by arsenic via Nrf2 signaling. BioMed Res. Int. 2015. [CrossRef] [PubMed]

31. Wu, W.; Li, Y.; Wu, Y.; Zhang, Y.; Wang, Z.; Liu, X. Lutein suppresses inflammatory responses through Nrf2 activation and NF-kB inactivation in lipopolysaccharide-stimulated BV-2 microglia. Mol. Nutr. Food Res. 2015, 59, 1663-1673. [CrossRef] [PubMed]

32. Havaux, M.; Dall'Osto, L.; Bassi, R. Zeaxanthin has enhanced antioxidant capacity with respect to all other xanthophylls in arabidopsis leaves and functions independent of binding to psii antennae. Plant Physiol. 2007, 145, 1506-1520. [CrossRef] [PubMed]

33. Perez-Galvez, A.; Viera, I.; Roca, M. Chemistry in the bioactivity of chlorophylls: An overview. Curr. Med. Chem. 2017, 24, 4515-4536. [CrossRef] [PubMed]

34. Fattore, M.; Montesano, D.; Pagano, E.; Teta, R.; Borrelli, F.; Mangoni, A.; Seccia, S.; Albrizio, S. Carotenoid and flavonoid profile and antioxidant activity in "Pomodorino vesuviano" tomatoes. J. Food Compos. Anal. 2016, 53, 61-68. [CrossRef]

35. Criddle, D.N.; Gillies, S.; Baumgartner-Wilson, H.K.; Jaffar, M.; Chinje, E.C.; Passmore, S.; Chvanov, M.; Barrow, S.; Gerasimenko, O.V.; Tepikin, A.V.; et al. Menadione-induced reactive oxygen species generation via redox cycling promotes apoptosis of murine pancreatic acinar cells. J. Biol. Chem. 2006, 281, 40485-40492. [CrossRef] [PubMed]

36. Bresgen, N.; Eckl, P.M. Oxidative stress and the homeodynamics of iron metabolism. Biomolecules 2015, 5, 808-847. [CrossRef] [PubMed]

37. Bystrom, L.M.; Guzman, M.L.; Rivella, S. Iron and reactive oxygen species: Friends or foes of cancer cells? Antioxid. Redox Signal. 2014, 20, 1917-1924. [CrossRef] [PubMed]

38. Odongo, G.A.; Schlotz, N.; Baldermann, S.; Neugart, S.; Ngwene, B.; Schreiner, M.; Lamy, E. Effects of Amaranthus cruentus L. on aflatoxin B1- and oxidative stress-induced DNA damage in human liver (HepG2) cells. Food Biosci. 2018, 26, 42-48. [CrossRef]

39. Xiao, L.; Luo, G.; Tang, Y.; Yao, P. Quercetin and iron metabolism: What we know and what we need to know. Food Chem. Toxicol. 2018, 114, 190-203. [CrossRef] [PubMed]

40. Murakami, M.; Yamaguchi, T.; Takamura, H.; Atoba, T.M. Effects of thermal treatment on radical-scavenging activity of single and mixed polyphenolic compounds. J. Food Sci. 2004, 69, FCT7-FCT10. [CrossRef]

41. Fiol, M.; Weckmuller, A.; Neugart, S.; Schreiner, M.; Rohn, S.; Krumbein, A.; Kroh, L.W. Thermal-induced changes of kale's antioxidant activity analyzed by HPLC-UV/Vis-online-TEAC detection. Food chem. 2013, 138, 857-865. [CrossRef] [PubMed]

42. De Beer, D.; Joubert, E.; Marais, J.; Manley, M. Unravelling the total antioxidant capacity of pinotage wines: Contribution of phenolic compounds. J. Agric. Food Chem. 2006, 54, 2897-2905. [CrossRef] [PubMed]

43. Oszmianski, J.; Kolniak-Ostek, J.; Wojdylo, A. Characterization of phenolic compounds and antioxidant activity of Solanum scabrum and Solanum burbankii berries. J. Agric. Food Chem. 2014, 62, 1512-1519. [CrossRef] [PubMed]

44. Sharma, T.; Airao, V.; Panara, N.; Vaishnav, D.; Ranpariya, V.; Sheth, N.; Parmar, S. Solasodine protects rat brain against ischemia/reperfusion injury through its antioxidant activity. Eur. J. Pharmacol. 2014, 725, 40-46. [CrossRef] [PubMed]

45. Hameed, A.; Ijaz, S.; Mohammad, I.S.; Muhammad, K.S.; Akhtar, N.; Khan, H.M.S. Aglycone solanidine and solasodine derivatives: A natural approach towards cancer. Biomed. Pharmacother. 2017, 94, 446-457. [CrossRef] [PubMed]

46. Kalalinia, F.; Karimi-Sani, I. Anticancer properties of solamargine: A systematic review. Phytother. Res. 2017, 31, 858-870. [CrossRef] [PubMed]

47. Hassenberg, K.; Huyskens-Keil, S.; Herppich, W.B. Impact of postharvest UV-C and ozone treatments on microbiological properties of white asparagus (Asparagus offificinalis L.). J. Appl. Bot. Food Qual. 2012, 85, 174-181.

48. Urban, L.; Charles, F.; de Miranda, M.R.A.; Aarrouf, J. Understanding the physiological effects of UV-C light and exploiting its agronomic potential before and after harvest. Plant Physiol. Biochem. 2016, 105, 1-11. [CrossRef] [PubMed] 
49. Huyskens-Keil, S.; Hassenberg, K.; Herppich, W.B. Impact of postharvest UV-C and ozone treatment on textural properties of white asparagus (Asparagus officinalis L.). J. Appl. Bot. Food Qual. 2011, 84, 229-234.

50. Jansen, M.A.K.; van den Noort, R.E.; Tan, M.Y.A.; Prinsen, E.; Lagrimini, L.M. Thorneley RNF. Phenol-oxidizing peroxidases contribute to the protection of plants from ultraviolet radiation stress. Plant Physiol. 2001, 126, 1012-1023. [CrossRef] [PubMed]

51. Schreiner, M.; Mewis, I.; Huyskens-Keil, S.; Jansen, M.A.K.; Zrenner, R.; Winkler, J.B.; O’Brien, N.; Krumbein, A. UV-B-induced secondary plant metabolites-potential benefits for plant and human health. CRC Crit. Rev. Plant Sci. 2012, 31, 229-240. [CrossRef]

52. Wilkening, S.; Stahl, F.; Bader, A. Comparison of primary human hepatocytes and hepatoma cell line Hepg2 with regard to their biotransformation properties. Drug Metab. Dispos. 2003, 31, 1035-1042. [CrossRef] [PubMed]

53. Gogo, E.O.; Opiyo, A.M.; Hassenberg, K.; Ulrichs, C.; Huyskens-Keil, S. Postharvest UV-C treatment for extending shelf life and improving nutritional quality of African indigenous leafy vegetables. Postharvest Biol. Technol. 2017, 129, 107-117. [CrossRef]

54. Ercolini, D.; Fogliano, V. Food design to feed the human gut microbiota. J. Agric. Food Chem. 2018, 66, 3754-3758. [CrossRef] [PubMed]

(C) 2018 by the authors. Licensee MDPI, Basel, Switzerland. This article is an open access article distributed under the terms and conditions of the Creative Commons Attribution (CC BY) license (http://creativecommons.org/licenses/by/4.0/). 